Supplementum Epigraphicum Graecum. Amsterdam 1923 ff. [SEG]

Sylloge Inscriptionum Graecarum. Ed. W. DitTENBERGER. Hildesheim 1960. [SIG]

Sylloge Nummorum Graecorum. Ed. Hans v. AuLock, Berlin 1964-1966. [SNG]

Tituli Asiae Minoris II. Pamphylia. Ed. Ernst KalinKA, Wien 1944. [TAM II]

Tolstol, Jean: Monnaies Byzantines. St. Petersburg 1912-1914, ND Amsterdam 1968 (russ.).

TOMASCHITZ, Kurt: Unpublizierte Inschriften Westkilikiens aus dem Nachlaß Terence B. Mitfords (= Österreichische AdW, phil.-hist. Klasse, Denkschriften 264, ETAM 21). Wien 1998.

Wrotн, Warwick: Catalogue of the Coins of the Vandals, Ostrogoths and Lombards, and of the Empires of Thessalonica, Nicaea and Trebizond, in the British Museum. London 1911.

ZACOS, Georges / VeGLeRY, Alexander: Byzantine Lead Seals I. Basel 1972.

\title{
3. Nachschlagewerke, Quellenkunden, Atlanten und Bibliographien
}

L'Année Philologique. Bibliographie critique et analystique de l'Antiquité Gréco-Latine. Fondée par Jean Marouzeau. Paris $1928 \mathrm{ff}$.

Assemanus, Ioseph Simonius / Evodius, Stephanus: Bibliothecae Apostolicae Vaticanae codicum manuscriptorum catalogus in tres partes distributus. 1. Teil Bd. 3 Rom 1759, ND Paris 1926.

Bagnall, Roger S. / Cameron, Alan / SchwarTZ, Seth R. / Worp, Klaas A.: Consuls of the Later Roman Empire (= Philological Monographs of the American Philological Association 36). Atlanta, Georgia 1987. [CLRE].

BAUER, Walter: Griechisch-deutsches Wörterbuch zu den Schriften des Neuen Testaments und der frïhchristlichen Literatur. Berlin-New York ${ }^{6} 1988$.

Bickerman, Elias J.: Chronology of the Ancient World. London ${ }^{2} 1980$.

Degrassi, Attilio: I fasti consolari dell' Impero romano dal 30 avanti cristo al 613 dopo Cristo. Rom 1952.

Dictionnaire d'Archéologie Chrétienne et de Liturgie. Paris 1924 ff. [DACL]

Dictionnaire d'Histoire et de Géographie Ecclésiastiques. Paris 1912 ff. [DHGE]

Hammond, Nicholas G. L.: Atlas of the Greek and Roman World in Antiquity. Park Ridge, NJ 1981.

JusTI, Ferdinand: Iranisches Namenbuch. Marburg 1895.

Karayannopoulos, Johannes / WeISS, Günther: Quellenkunde zur Geschichte von Byzanz (3241453), 2 Bde. Wiesbaden 1982. [KW]

KIENAST, Dietmar: Römische Kaisertabelle. Grundzüge einer römischen Kaiserchronologie. Darmstadt ${ }^{2} 1996$.

Late Antiquity. A Guide to the postclassical World. Hrsg. v. Glen W. Bowersock / Peter Brown / Oleg Grabar. Cambridge, Mass.-London 1999.

Lexikon der Alten Welt. Zürich - Stuttgart 1965. [LAW]

Lexikon fir Theologie und Kirche. Freiburg i.Br. ${ }^{2} 1957-21968 ;{ }^{3} 1993-{ }^{32} 2001$. [LThK]

LidDELL, Henry G. / SCOTT, Robert: A Greek-English Lexicon. With a revised supplement. Oxford 1996. 
Mason, Hugh J.: Greek Terms for Roman Institutions. A Lexicon and Analysis (= American Studies in Papyrology 13). Toronto 1974.

MoravcsIK, Gyula: Byzantoturcica I. Die byzantinischen Quellen der Geschichte der Turkvölker (= Berliner Byzantinische Arbeiten 10). Berlin ${ }^{2} 1958$.

MUELleR-WIENER, Wolfgang u.a.: Bildlexikon zur Topographie Istanbuls. ByzantionKonstantinopolis-Istanbul bis zum Beginn des 17. Jahrhunderts. Tübingen 1977.

Der Neue Pauly. Hrsg. Hubert CANCIK und Helmut SCHNEIDER. Stuttgart-Weimar 1996-2003. $[D N P]$

The Oxford Dictionary of Byzantium. Ed. Alexander P. KaZHDan. Oxford University Press 1991. $[O D B]$

The Princeton Encyclopedia of Classical Sites, ed. R. Stillwell / W. L. MacDonald / M. H. MCALlister. Princeton, New Jersey 1976.

Prosopographia Imperii Romani Saec. I. II. III. Editio Altera, ed. Preußische / BerlinBrandenburgische AdW. Berlin 1933 ff. [PIR]

The Prosopography of the Later Roman Empire, Vol. I: Arnold H. M. Jones / John R. Martindale / John Morris, A.D. 260-395. Cambridge 1971; John R. Martindale, 'Prosopography of the Later Roman Empire: addenda et corrigenda to Volume I', in: Historia 29 (1980), 474 497; Vol. II: John R. Martindale, A.D. 395-527. Cambridge 1980; Vol. III A/B: John R. Martindale, A.D. 527-641. Cambridge 1992 [PLRE]; Ralph W. Mathisen, 'A Survey of Significant Addenda to P.L.R.E.' in: Medieval Prosopography 8/1 (1987), 5-30.

Prosopographie der mittelbyzantinischen Zeit. Ed. Ralph-Johannes LILIE / Claudia LudwIG / Thomas Pratsch / Ilse Rochow u.a., 1. Abteilung. 7 Bde. Berlin 1998-2001. [PmbZ]

Paulys Realencyclopädie der classischen Altertumswissenschaft. München 1893-1986. [RE]

Reallexikon für Antike und Christentum. Stuttgart $1950 \mathrm{ff}$. [RAC]

Reallexikon zur byzantinischen Kunst Bd. 4. Stuttgart 1990.

ROSCHER, Wilhelm H. (Hrsg.): Ausführliches Lexikon der griechischen und römischen Mythologie. Leipzig 1909-1915.

SonNABEND, Holger (Hrsg.): Mensch und Landschaft in der Antike. Lexikon der Historischen Geographie. Stuttgart-Weimar 1999.

Thomasson, Bengt E.: Laterculi Praesidum Vol. I. Göteborg 1984.

Tübinger Atlas des Vorderen Orients. Ed. Sonderforschungsbereich 19. TAVO. Tübingen $1977 \mathrm{ff}$. [TAVO]

Zgusta, Ladislav: Kleinasiatische Personennamen. Prag 1964. 